\title{
Desempenho Técnico em Equipes de Voleibol Sentado Masculino
}

\author{
Technical Performance in Sitting Men's Volleyball teams
}

Andrew Carneiro Borges ${ }^{\mathrm{a}}$; Leonardo dos Santos Oliveira ${ }^{\mathrm{b}}$; Antônio Meira Neto ${ }^{\mathrm{a}}$; Gilmário Ricarte Batista ${ }^{* *}$; Elaine Cappellazzo Souto ${ }^{\text {ad }}$

\author{
${ }^{a}$ Universidade Federal da Paraíba. Departamento de Educação Física. João Pessoa, Paraíba.

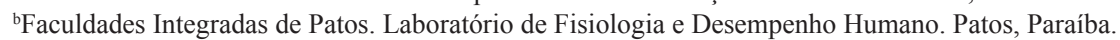 \\ 'Universidade Federal da Paraíba. Programa de Pós-Graduação Stricto Sensu em Educação Física. João Pessoa, Paraíba. \\ dUniversidade Estadual de Londrina, Programa de Pós-Graduação Stricto Sensu em Educação Física \\ *E-mail: cajagr@gmail.com \\ Recebido em: 09/09/2015; Aceito em: 30/10/2015
}

\begin{abstract}
Resumo
A análise do jogo consiste em uma das estratégias para o técnico identificar os fatores que interferem no rendimento de suas equipes. O presente estudo teve como objetivo comparar o desempenho técnico do saque e ataque no voleibol sentado entre equipes finalistas de uma competição da Região Nordeste. Participaram do estudo quatro equipes do Campeonato Alagoano de Voleibol Sentado Masculino de 2014. Foram filmados seis jogos e analisados 522 saques e 267 ataques. A análise de jogo foi realizada por meio de uma escala likert de cinco pontos e o desempenho técnico foi avaliado por coeficientes de performance de saque (CPS) e ataque (CPA). Para comparar o desempenho entre as equipes foi utilizada a ANOVA one-way, com post hoc de Tukey, com significância de $5 \%$. Os resultados revelaram uma tendência linear no $\operatorname{CPS}\left(1^{\circ}\right.$ lugar $=2,47 \pm 0,30 ; 2^{\circ}$ lugar $=2,09 \pm 035 ; 3^{\circ}$ lugar $=2,04 \pm 0,35 ; 4^{\circ}$ lugar $\left.=1,57 \pm 0,35\right)$, mas foi encontrada diferença significativa apenas entre o $1^{\circ}$ lugar e o $2^{\circ}$ lugar $(p=0,02)$. Em adição, não foram encontradas diferenças estatísticas no CPA $\left(1^{\circ} \operatorname{lugar}=2,13 \pm 0,31 ; 2^{\circ} \operatorname{lugar}=21,66 \pm 055 ; 3^{\circ}\right.$ lugar $=1,87 \pm 0,33 ; 4^{\circ}$ lugar $=2,38 \pm 1,06$ ). Concluiu-se que o saque foi um fator determinante para o sucesso na competição de voleibol sentado e o ataque pode variar.
\end{abstract}

Palavras-chave: Voleibol. Pessoas com Deficiência. Desempenho Atlético.

\begin{abstract}
Match analysis consists in a strategy used for the coach to identify the factors that affect the performance of its teams. This study aimed to compare the technical performance of serve and attack on the sitting volleyball teams among finalists in a competition of the Northeast region. Study participants were four teams of the Volleyball Sitting Male Alagoas Championship 2014. They were filmed six games and analyzed 522 serves and 267 attacks. The game analysis was performed using a Likert scale of five points, the technical performance was evaluated by performance serve coefficients (PSC) and attack (PAC). To compare performance between the teams it was used one-way ANOVA with post hoc Tukey, with 5\% significance. The results revealed a linear trend in the PCS $\left(1^{\text {st }}\right.$ place $=2.47 \pm 0.30 ; 2^{\text {nd }}=2.09 \pm 035 ; 3^{\text {rd }}=2.04 \pm 0.35 ; 4^{\text {th }}$ place $=1.57 \pm 0.35)$, but it was found a significant difference only between the $1^{\text {st }}$ place and the $2^{\text {nd }}(p=0.02)$. In addition, there were no statistical differences in PAC (1 $1^{\text {st }}$ place $=2.13 \pm 0.31 ; 2^{\text {nd }}=21.66 \pm 055 ; 3^{\text {rd }}=1.87 \pm 0.33 ; 4^{\text {th }}$ place $\left.=2.38 \pm 1.06\right)$. It was concluded that the serving was a determining factor for success in the competition in sitting volleyball, and the attack can vary.
\end{abstract}

Keywords: Volleyball. Disabled Persons. Athletic Performance.

\section{Introdução}

O voleibol, desde sua criação, tem demonstrado grande evolução nos aspectos técnico, tático e físico ${ }^{1}$. Nesse sentido, técnicos de vários clubes têm buscado estratégias para a melhoria da performance de suas equipes, dentre elas, a análise do jogo. Geralmente, a análise do jogo engloba as fases de observação dos acontecimentos, registro dos dados e posterior interpretação ${ }^{2}$. Segundo Garganta ${ }^{3}$, a pesquisa nesse âmbito utiliza-se de diferentes denominações, como observação do jogo, análise do jogo e análise notacional.

A informação obtida pela análise do comportamento de atletas em jogos é considerada uma das variáveis que mais afetam a aprendizagem e a eficácia da ação desportiva ${ }^{4}$. Por isso, o conhecimento acerca da competência com que os jogadores e as equipes realizam as diferentes ações têm-se revelado fundamental para melhorar significativamente as técnicas dos jogadores em relação às ações de jogo ${ }^{5}$.

Os jogos de voleibol de alto nível serviram de inspiração para estudar a tendência de uma ação específica, por exemplo o ataque, ou para uma análise detalhada da técnica e da tática empregada em todos os fundamentos ${ }^{6}$. Além disso, alguns estudos também se dedicam a analisar as relações entre as ações do jogo de voleibol e o resultado da partida ${ }^{7}$.

Uma investigação prévia realizada por Moutinho et al. ${ }^{8}$ verificou, por meio da análise da dinâmica do jogo, que os métodos ofensivos se mostram como determinantes do sucesso no voleibol, sendo então o ataque uma ação prioritária do jogo. Corroborando com este pressuposto, Rocha e Barbanti ${ }^{7}$ observaram grande influência da qualidade da recepção do saque na ofensividade do ataque.

Assim como no voleibol convencional, a tática que 
corresponde ao comportamento adotado por uma equipe durante o jogo, individual ou coletivo, necessita de bom desempenho técnico dos jogadores ${ }^{9}$, sendo, também, essencial no voleibol sentado. Na parte técnica, além da execução dos fundamentos básicos de saque, recepção, levantamento, ataque, bloqueio e defesa, o deslocamento realizado sentado, no movimento pelo solo por meio das mãos ${ }^{10}$ é a principal diferença desse esporte.

Como jogo coletivo, o voleibol sentado é uma das modalidades que tem adquirido muitos adeptos no Brasil e no mundo. Comparada a outras modalidades coletivas paralímpicas, como basquetebol e rugby em cadeira de rodas, exige pouco investimento financeiro para sua implantação e um perfil de atletas com deficiência física mais autônomos, sendo em sua maioria amputados. Contudo, pesquisas com o voleibol sentado ainda são escassas, especialmente no que se refere à análise do jogo, por exemplo; já foram investigadas a caracterização do levantamento ${ }^{11}$, o deslocamento e a predominância dos fundamentos ${ }^{12}$, a determinação temporal do jogo ${ }^{13}$ e os indicadores de desempenho ${ }^{14}$. Diante disso, observou-se que existem lacunas do conhecimento no que concerne ao desempenho técnico no voleibol sentado.

Portanto, tendo em vista a importância da análise do jogo de voleibol para a melhoria da performance dos atletas e das equipes, o presente estudo teve como objetivo comparar o desempenho técnico do saque e ataque no voleibol sentado entre as equipes finalistas de uma competição da Região Nordeste.

\section{Material e Métodos}

Este estudo distingue-se como descritivo observacional, com delineamento transversal. Segundo Thomas et al. ${ }^{15}$, a pesquisa descritiva observacional caracteriza-se pela observação do comportamento de determinados sujeitos em seu ambiente natural, podendo, assim, ser realizado em um único momento do tempo ${ }^{16}$. O trabalho teve a aprovação de um comitê de ética local (Parecer n ${ }^{\circ}$ 637.957/2014), uma vez que o estudo se encontrava em consonância com o disposto na Resolução no 466/12 do Conselho Nacional de Saúde.

Fizeram parte do estudo quatro equipes masculinas de voleibol sentado, participantes do Campeonato Alagoano de Voleibol Sentado. Analisou-se a disputa de seis partidas, sendo contabilizadas 522 ações de saque e 267 ações de ataque. Todas as informações obtidas foram registradas em uma planilha desenvolvida pelos pesquisadores, constando: número de ações, identificação da equipe e dos jogadores, ações técnico-táticas e a qualidade de execução.

Para coleta das informações, utilizaram-se duas câmeras semiprofissionais (Sony, EUA) posicionadas no fundo da quadra, a fim de permitir a observação de todas as ações dos jogos (20 metros da quadra), facilitando, desta maneira, a análise dos saques e ataques. As filmagens foram analisadas por um único avaliador com experiência em avaliação e scout técnico, que analisou os fundamentos seguindo os critérios adotados em outros estudos ${ }^{17,18}$. Em seguida, os dados registrados na planilha foram tabulados no programa Microsoft Excel 2010 para que fossem calculados os coeficientes de saque e ataque.

Os critérios propostos por Eom e Schutz ${ }^{17}$ (Quadro 1) sugerem que uma habilidade no voleibol pode ser avaliada qualitativamente dentro de uma escala Likert que varia de "zero" a "quatro"13. O desempenho técnico foi avaliado por meio dos coeficientes de performance ${ }^{18}$, sendo o coeficiente de performance de saque (CPS) e o coeficiente de performance de ataque (CPA) calculados pela equação de ações terminais, dada por: Coeficiente de performance $=[4 \times$ (ações de pontos $)$ $+2 \times$ (ações de continuidade) $] \div$ [Total de ações].

Quadro 1: Avaliação das ações do saque e ataque

\begin{tabular}{|l|l|}
\hline & Saque \\
\hline Escore 0 & $\begin{array}{l}\text { Erro que resulta na perda do ponto direto para a } \\
\text { equipe adversária. }\end{array}$ \\
\hline Escore 1 & $\begin{array}{l}\text { Saque que facilita a ação da recepção do adversário, } \\
\text { possibilitando uma organização ofensiva. }\end{array}$ \\
\hline Escore 2 & $\begin{array}{l}\text { Saque que dificulta a ação da recepção da equipe } \\
\text { adversária, mas possibilitando uma organização } \\
\text { ofensiva limitada. }\end{array}$ \\
\hline Escore 3 & $\begin{array}{l}\text { Saque que dificulta a recepção da equipe adversária, } \\
\text { mas não possibilitando uma organização ofensiva. }\end{array}$ \\
\hline Escore 4 & $\begin{array}{l}\text { Saque que permite marcar um ponto direto na equipe } \\
\text { adversária. }\end{array}$ \\
\hline Escore 0 & $\begin{array}{l}\text { Ataque } \\
\text { equipe adversária. }\end{array}$ \\
\hline Escore 1 1 & $\begin{array}{l}\text { Ataque que facilita a ação defensiva do adversário, } \\
\text { possibilitando uma organização ofensiva de contra- } \\
\text { ataque. }\end{array}$ \\
\hline Escore 2 & $\begin{array}{l}\text { Ataque que dificulta a ação defensiva do adversário, } \\
\text { mas possibilitando uma organização ofensiva de } \\
\text { contra-ataque limitado. }\end{array}$ \\
\hline $\begin{array}{l}\text { Ataque que dificulta a ação defensiva, mas não } \\
\text { possibilita uma organização ofensiva de contra- } \\
\text { ataque ou quando a bola é tocada pelo bloqueio } \\
\text { e permanece na mesma quadra do atacante, } \\
\text { possibilitando um novo ataque. }\end{array}$ \\
\hline $\begin{array}{l}\text { Ataque que permite marcar um ponto direto na } \\
\text { equipe adversária. }\end{array}$ \\
\hline
\end{tabular}
Fonte: Dados da pesquisa.

A análise dos dados foi realizada no programa estatatístico SPSS 22.0 (IBM SPSS Inc., EUA). Para verificar a normalidade dos dados foi realizado o teste de ShapiroWilk e a homogeneidade pelo teste de Levene. A estatística descritiva foi reportada em média, desvio padrão, distribuição de frequência e percentual. Para comparar diferenças no desempenho técnico entre as equipes finalistas do Campeonato Alagoano de Voleibol Sentado foi utilizado o teste da ANOVA one-way, quando encontradas diferenças significativas foi utilizado o teste de Tukey. Para as análises foram consideradas significância de 5\%. 


\section{Resultados e Discussão}

O Quadro 2 reporta os valores dos escores da análise de jogo. A equipe que ficou em primeiro lugar na competição apresentou os maiores valores percentuais na escala likert tanto no saque $(30,66 \%)$ quanto no ataque $(50,59 \%)$.

Quadro 2: Distribuição de frequência e percentual dos escores do saque e ataque de acordo com as equipes finalistas do campeonato alagoano de voleibol sentado

\begin{tabular}{|c|c|c|c|c|}
\hline Fundamento & $\begin{array}{c}\mathbf{1}^{\mathbf{0}} \text { lugar } \\
\text { Frequência } \\
\mathbf{( \% )}\end{array}$ & $\begin{array}{c}\mathbf{2}^{\mathbf{0}} \text { lugar } \\
\text { Frequência } \\
\mathbf{( \% )}\end{array}$ & $\begin{array}{c}\mathbf{3}^{\mathbf{o}} \text { lugar } \\
\text { Frequência } \\
\mathbf{( \% )}\end{array}$ & $\begin{array}{c}\mathbf{4}^{\mathbf{0}} \text { lugar } \\
\text { Frequência } \\
(\mathbf{\%})\end{array}$ \\
\hline Saque & & & & \\
\hline 0 & $24(16,00)$ & $13(10,66)$ & $21(20,79$ & $19(12,75)$ \\
\hline 1 & - & - & - & - \\
\hline 2 & $49(32,67)$ & $68(55,74)$ & $45(44,55)$ & $82(55,03)$ \\
\hline 3 & $31(20,67)$ & $22(18,03)$ & $21(20,79)$ & $25(16,78)$ \\
\hline 4 & $46(30,66)$ & $19(15,57)$ & $14(13,86)$ & $23(15,44)$ \\
\hline Ataque & & & & \\
\hline 0 & $23(27,06)$ & $16(20,79)$ & $6(12,77)$ & $8(13,79)$ \\
\hline 1 & $14(16,47)$ & $24(31,17)$ & $10(21,28)$ & $14(24,14)$ \\
\hline 2 & $2(3,53)$ & $4(5,19)$ & $8(17,02)$ & $13(22,41)$ \\
\hline 3 & $3(3,53)$ & $6(7,79)$ & $4(8,51)$ & $1(1,72)$ \\
\hline 4 & $43(50,59)$ & $27(35,06)$ & $19(40,42)$ & $22(37,94)$ \\
\hline
\end{tabular}

Fonte: Dados da pesquisa.

O Quadro 3 compara os coeficientes de performance do saque e do ataque entre as equipes participantes do Campeonato Alagoano de Voleibol Sentado. Com base na análise dos coeficientes, verificou-se que houve superioridade da equipe campeã sobre o último colocado apenas para o CPS $(\mathrm{p}=0,02)$.

Quadro 3: Comparação do desempenho técnico do saque e ataque entre as equipes finalistas do campeonato alagoano de voleibol sentado

\begin{tabular}{|l|c|c|c|c|c|c|}
\hline Variável & $\mathbf{1}^{\mathbf{0}}$ lugar & $\mathbf{2}^{\mathbf{0}}$ lugar & $\mathbf{3}^{\mathbf{0}}$ lugar & $\mathbf{4}^{\mathbf{0}}$ lugar & $\mathbf{F}$ & $\mathbf{P}$ \\
\hline CPS & $2,47 \pm 0,30$ & $2,09 \pm 0,35$ & $2,04 \pm 0,35$ & $1,57 \pm 0,35^{*}$ & 6,343 & 0,02 \\
\hline CPA & $2,13 \pm 0,31$ & $1,66 \pm 0,55$ & $1,87 \pm 0,33$ & $2,38 \pm 1,06$ & 0,706 & 0,58 \\
\hline
\end{tabular}
Valores reportados por média e desvio padrão. CPS $=$ Coeficiente de performance de saque; $\mathrm{CPA}=$ Coeficiente de performance de ataque. ${ }^{*} \mathrm{P}<$ 0,05 ; Diferença significativa entre o $1^{\circ}$ lugar vs. $4^{\circ}$ lugar.

Fonte: Dados da pesquisa.

A tendência linear verificada no CPS entre o primeiro e o quarto lugar na competição no presente estudo, corrobora que o saque é um determinante no voleibol sentado. Sabese que a agressividade do saque está integrada com a redução das possibilidades de ataque da equipe adversária e com o aumento das defesas da própria equipe ${ }^{19}$. Um estudo prévio de Katsikadelli ${ }^{20}$ demonstra que o fundamento saque proporcionou uma quantidade significativamente maior de erros de recepção da equipe adversária, confirmando que a eficácia do saque afeta, diretamente, o rendimento das ações de recepção e as opções de ataque da equipe adversária. Em outra pesquisa, Costa et al. ${ }^{21}$ relatam que, além de o saque ter proporcionado condições não favoráveis ao ataque adversário, gerou fragilidades ao nível de organização ofensiva do adversário, situação que merece maior atenção no processo de treinamento esportivo da modalidade. Nesse sentido, a melhor forma de recepcionar o saque no voleibol sentado é por meio do toque ${ }^{11}$.

No presente estudo, verificou-se a ausência de diferença significativa no ataque entre as equipes. Isto talvez possa ser justificado pelo fato de o fundamento ataque ser precedido por outros fundamentos que não foram analisados neste estudo, como a recepção do saque e o levantamento, constituindo uma limitação da presente investigação. No voleibol de alto rendimento, as recepções de "baixa" qualidade promovem menor eficácia no ataque, ao passo que as de "alta" qualidade promovem exatamente o contrário, confirmando assim, a grande influência da qualidade da recepção do saque sobre a ofensividade do ataque ${ }^{7}$.

Bayer $^{22}$ verificou que quando a recepção era ruim, cerca de $49 \%$ dos ataques proporcionavam que as bolas continuassem em jogo, com vantagem para a equipe que estava na defensiva. Por outro lado, quando a recepção era média, boa ou perfeita, de $47 \%$ a $62 \%$ dos ataques ocasionavam pontos. Estes resultados indicam a influência da qualidade da recepção na execução do ataque. $\mathrm{O}$ fato de o saque adversário induzir menor ocorrência de ponto no ataque sugere a fraqueza na organização ofensiva da equipe diante das dificuldades proporcionadas por uma boa execução deste ${ }^{21}$. A recepção do saque é o primeiro fundamento a ser realizado, possibilitando assim, a organização ofensiva da equipe no voleibol.

Com base no exposto, pode-se afirmar que o saque, quando bem executado, torna-se o fundamento de maior importância no voleibol, tanto no convencional quanto no sentado, e que o ataque apesar de ser o fundamento de maior importância na ofensividade do jogo, depende da execução de outros fundamentos para ser efetivamente decisivo em um jogo.

\section{Conclusão}

Pode-se concluir que entre as equipes analisadas no voleibol sentado o desempenho técnico do saque foi determinante para o sucesso na competição, pois mesmo apresentando diferença significativa somente entre o primeiro e o quarto lugar, também percebeu-se que a equipe primeira colocada apresentou no CPS valores superiores entre o primeiro e os outros colocados apesar de não apresentar diferença estatisticamente. Em relação ao ataque, ele pode variar independentemente da colocação da equipe. A produção de mais estudos pode subsidiar referências para treinadores e jogadores como um dos pontos chave em busca da excelência esportiva.

\section{Referências}

1. Rocha MA, Barbanti JB. Análise das ações de saltos de ataque, bloqueio e levantamento no voleibol feminino. Rev Bras Cineantropom Desempenho Hum 2007;9(3):284-90.

2. Hughes M. Notational Analysis. In: Reilly T, Williams AM. Science and soccer. London: Routledge; 2003. p.343-61. 
3. Garganta J. Modelação táctica do jogo de futebol: estudo da organização da fase ofensiva em equipas de alto rendimento. Porto. Tese [Doutorado em Ciências do Desporto] Universidade do Porto; 1997.

4. Hughes MD, Franks IM. Notational analysis of sport. London: E\&FN Spon; 1997.

5. Garganta J. A análise da performance nos jogos desportivos: revisão acerca da análise do jogo. Rev Port Ciênc Desporto 2001;1(1):57-64.

6. Gouvea FL. Análise das ações de jogos de voleibol e suas implicações para o treinamento técnico-tático da categoria infanto-juvenil feminina (16 e 17 anos). Campinas. Dissertação [Mestrado em Educação Física] - Universidade Estadual de Campinas; 2004.

7. Rocha CM, Barbanti VJ. Uma análise dos fatores que influenciam o ataque no Voleibol masculino de alto nível. Rev Bras Educ Fís Esp 2004;18(4):303-14.

8. Moutinho C, Marques A, Maia J. Estudo da estrutura interna das acções da distribuição em equipes de voleibol de alto nível de rendimento. In: Mesquita I, Moutinho C, Faria R. Investigação em voleibol: estudos ibéricos. Porto: FCDEFUP; 2003. p.107-29.

9. Zerger M. A study of movement in sitting-volleyball. Oklahoma. Dissertação [Mestrado em Ciência] - University of Central Oklahoma; 2008.

10. Carvalho CL, Gorla JI, Araújo PF. Voleibol sentado: do conhecimento à iniciação da prática. Conexões 2013;11(2);97126.

11. Macedo CD, Mesquita I, Silva MA. Análise das características da recepção e do levantamento no jogo de voleibol sentado de alto nível. Rev Sobama 2007;12(1):21-5.

12. Apontes D, Santos EP, Veloso GHD, Silva RP, Freitas A.
Análise descritiva das ações de deslocamento e fundamentos do voleibol sentado. ConScientiae Saúde 2014;13:63-5.

13. Paulo AC, Kitamura KRP, Paula RO, Ferreira Junior ON, Oliveira R, Gimenez R. Tempo de rally no voleibol sentado: recursos para programas de treinamento ConScientiae Saúde 2014;13:70-3.

14. Haiachi MC, Oliveira BRR, Almeida MB, Santos TM. Indicadores de desempenho no voleibol sentado. Rev Educ Fís/UEM 2014;25(3):335-43.

15. Thomas JR, Nelson JK, Silverman SJ. Métodos de pesquisa em atividade física. Porto Alegre: Artmed; 2012.

16. Sousa VD, Driessnack M, Mendes IAC. Revisão dos desenhos de pesquisa relevantes para enfermagem. Parte 1: desenhos de pesquisa quantitativa. Rev Latinoam Enferm 2007;15(3):1-6.

17. Eom HJ, Schutz RW. Statistical analysis of volleyball team performance. Res Q Exerc Sport 1992;63(1):11-8.

18. Marcelino R, Mesquita I, Sampaio J, Moraes JC. Estudo dos indicadores de rendimento em voleibol em função do resultado do set. Rev Bras Educ Fís Esporte 2010;24(1):6978.

19. Rocha CM, Barbanti VJ. An analysis of the confrontations in the first sequence of game actions in Brazilian volleyball. J Hum Mov Stud 2006;50(4):259-72.

20. Katsikadelli A. Reception and the attack serve of the world's leading volleyball teams. J Hum Mov Stud 1998;34:223-32.

21. Costa GCT, Mesquita I, Greco PJ, Ferreira NN, Morais JCL. Relação saque, recepção e ataque no voleibol juvenil masculino. Motriz Rev Educ Fís 2011;17(1):11-8.

22. Bayer C. La enseñanza de los juegos deportivos colectivos. Barcelona: Hispano Europea; 1986. 